\title{
Effects of trimetazidine therapy in patients with stable ischaemic heart disease
}

\author{
Dora Fabijanović*, Martina Lovrić Benčić, Tea Šimonček \\ University of Zagreb School of Medicine, University Hospital Centre Zagreb, Zagreb, Croatia
}

\begin{abstract}
Background: Current antianginal medications include betablockers and calcium antagonists, which decrease ischaemic severity by reducing cardiac workload, and nitrates, which increase coronary blood flow. Additional therapy with metabolic agents, such as trimetazidine, which shifts cardiac energy metabolism by inhibiting beta-oxidaton of fatty acids by blocking long-chain 3-ketoacyl-CoA thiolase, and which enhances glucose oxidation, may improve cardiac function during ischaemia.
\end{abstract}

Patients and Methods: We studied 82 patients (53 men and 29 women, mean age 61.6 years, SD 9.11) with ischaemic heart disease and left ventricular dysfunction who were followed-up at our outpatient department. All patients had either acute myocardial infarction and percutaneous transluminal coronary angioplasty, or aorto-coronary bypass. On study entry, all patients underwent echocardiography (EF $41.7 \%$, SD 11.55), cardiopulmonary exercise testing, and had 24 hour Holter monitoring. All of them received trimetazidine dihydrocloridum $35 \mathrm{mg}$ twice a day. After 6-12 months of follow-up, they repeated exercise testing, and

Received: $16^{\text {th }}$ Apr 2014

*Address for correspondence: Klinički bolnički centar Zagreb, Kišpatićeva 12, HR10000 Zagreb, Croatia.

Phone: +385-98-9387273

E-mail: dora.fabijanovic@gmail.com had 24 hour Holter monitoring. The results were compared according to diabetes, sex, age and ejection fraction.

Statistics: Wilcoxson rank test.

Results: All patients showed statistically significant $(p<$ 0.001 ) improvement in exercise testing (less pronounced ST depression) and lower number of ventricular premature beats (VPB) in Holter ECG. The results were the same for diabetics and nondiabetics, women and men equally, subgroups according to EF $(<40 \%, 40-50 \%,>50 \%)$ also showed improved results in exercise testing and less VPBs. There were also no differences among the subgroups according to age $(<65$, and $>65$ years), sex and diabetes, and age and diabetes.

Conclusion: The addition of trimetazidine $35 \mathrm{mg}$ twice a day to the patients with ischaemic heart disease resulted in greater improvements in functional capacity, better results in exercise testing, less ventricular premature beats regardless of age, sex, diabetes and ejection fraction. The effect of trimetazidine on glucose oxidation optimizes cellular energy processes, thereby maintaining proper energy metabolism during ischaemia. By preserving energy metabolism in cells exposed to hypoxia or ischaemia, trimetazidine prevents a decrease in intracellular ATP levels, thereby ensuring the proper functioning of ionic pumps and transmembrane sodium-potassium flow whilst maintaining cellular homeostasis, which is also indirectly confirmed by our results.

KEYWORDS: Ishaemic heart disease, trimetazidine, antianginal agents, exercise testing, Holter monitoring.

CITATION: Cardiol Croat. 2014;9(5-6):250. 